\title{
Monitoring electroconvulsive therapy by electroencephalogram: an update for ECT practitioners
}

\author{
Allan I. F. Scott
}

Abstract The purpose of this article is to update practitioners on the latest published research into the prevalence of prolonged cerebral seizure activity following electroconvulsive therapy (ECT). This research is drawing attention to the real practical challenges of recording and reading an electroencephalogram (EEG) tracing in the ECT clinic. In particular, determination of the seizure end-point is not always practicable and this poses a major problem in the detection and management of prolonged cerebral seizure activity. Some practical tips are suggested, and an update is given on the status of EEG monitoring in the assessment of seizure adequacy.

One of the tenets that influenced the Royal College of Psychiatrists' revised guidelines on electroconvulsive therapy (ECT) is the importance of minimising cognitive adverse effects (Scott, 2005). Prescribers are now encouraged in the initial use of unilateral ECT in illness that is not life-threatening, and a number of refinements to the administration of treatment have been recommended to ECT practitioners. One such refinement is that monitoring by electroencephalogram (EEG) must be available in all ECT clinics and that EEG monitoring is always carried out at least during the first treatment in a course (Royal College of Psychiatrists, 2005). The rationale is that EEG monitoring is the most direct method of detecting prolonged cerebral seizure activity, which increases the risk of adverse cognitive effects without any commensurate increase in clinical efficacy; the risk of prolonged cerebral seizure activity is greatest during the first treatment.

The revised College guidelines were completed when there were few data on the prevalence of prolonged cerebral seizures in contemporary practice. For example, there were no prospective studies from the UK on this topic. Two studies have since been published, and their findings are discussed here. We suggest some practical tips for addressing the challenges of EEG monitoring of cerebral seizures in the ECT clinic, and end our article with an update on the current status of EEG monitoring in the assessment of seizure adequacy. It is assumed the reader is already familiar with the section on monitoring seizure activity in the revised guidelines (Royal College of Psychiatrists, 2005).

\section{Prolonged cerebral seizures in clinical practice}

Benbow and colleagues (2003) have reported the prevalence of prolonged cerebral seizures in routine clinical practice in their ECT clinic in central Manchester. Cerebral seizures were monitored by a single-channel EEG and the seizure was considered prolonged if it lasted longer than $120 \mathrm{~s}$; the end of the seizure was determined by the computer built into the ECT machine. The prevalence of prolonged cerebral seizures was reported as the proportion of courses of ECT in which at least one such seizure was detected: EEG revealed at least one prolonged cerebral seizure in 18 out of 95 courses (19\%) given to 67 individual patients.

This reported prevalence is much higher than would have been anticipated by most ECT practitioners in the $\mathrm{UK}$, but is similar to the challenging findings from Bangalore (India) that had been the most pressing reason for the recommendation that monitoring by EEG must be available in all ECT clinics. Mayur et al (1999) had found that $16 \%$ of patients experienced prolonged cerebral seizure activity at the first administration of ECT, and in

Allan Scott is a consultant psychiatrist in general adult psychiatry and an honorary senior lecturer in the Andrew Duncan Clinic at the Royal Edinburgh Hospital (Morningside Terrace, Edinburgh EH10 5HF, UK. Email: Fiona.Morrison@lpct.scot.nhs.uk). He is a member of the Royal College of Psychiatrist's Special Committee on ECT and is the editor of the second edition of The ECT Handbook (Royal College of Psychiatrists, 2005). 
about one-third of cases this was detectable only by EEG monitoring - the convulsion itself was not prolonged.

When these findings from Manchester were published, a similar study was already underway in the ECT clinic at the Royal Edinburgh Hospital; the results have now been published (Whittaker $e t$ $a l, 2007)$. The aim was to establish the prevalence of prolonged cerebral seizures during the first treatment in a course of ECT, when the risk is greatest. In this study, cerebral seizures were monitored by two-channel EEG, one from each side of the head using a prefrontal mastoid positioning of the EEG electrodes. The end-point of the cerebral seizure was determined by the treating psychiatrist and not by the ECT machine's in-built computer. The EEG tracings were subsequently re-read by an independent rater, a consultant clinical neurophysiologist. Two definitions of prolonged cerebral seizure activity were used, one a minimum time of $120 \mathrm{~s}$ and the other a minimum time of $180 \mathrm{~s}$; the first definition was based on the College's revised guidelines and the second on the guidelines of the American Psychiatric Association (2001).

Only one cerebral seizure lasting as long as 180 s and only two lasting as long as $120 \mathrm{~s}$ were recorded in 100 individual patients during the first treatment in a course of ECT. The incidences of prolonged cerebral seizures were therefore $1 \%$ and $2 \%$, depending on which guideline was followed. The $95 \%$ confidence intervals (CIs) of these incidences did not extend to $16 \%$, let alone $19 \%$, suggesting that the differences between the observations from the Edinburgh ECT clinic and from the ECT clinics in Bangalore and Manchester did not simply arise by chance.

The detection of prolonged cerebral seizures is not just an academic matter. Although there is not yet a general consensus about the definition of a prolonged cerebral seizure, there is consensus that, once detected, such seizures should be terminated immediately by the intravenous administration of either more induction agent or a benzodiazepine drug (American Psychiatric Association, 2001; Royal College of Psychiatrists, 2005). These differences in the reported prevalences merit further consideration because of the clinical importance of the detection and management of prolonged cerebral seizures.

\section{Practical challenges to EEG recording in the ECT clinic}

One possible explanation for the differences in reported prevalences of prolonged cerebral seizures is variation in methods of EEG recording and analysis. There are substantial methodological problems in both, but discussion of these problems has been restricted to the specialist literature. Here I will focus on the methodological problems of determining the end of cerebral seizure activity; this and wider problems are well reviewed by Weiner et al (1991).

Weiner and colleagues stress that the determination of the end-point of a cerebral seizure is not as simple as the textbooks suggest. It can be straightforward in an EEG recording of regular paroxysmal discharges that end abruptly and are followed by sufficient post-ictal suppression to lead to a relatively flat recording after the cerebral seizure. They note that three problems can arise:

- the cerebral seizure activity may end not abruptly, but gradually

- the cerebral seizure may be followed by only limited, that is, relative post-ictal suppression

- the analysis of the EEG recording may be difficult or impossible because of the presence of artefact.

These problems can coexist, making determination of the seizure end-point impossible.

Figure 1 shows an example from my clinic of a cerebral seizure that ends abruptly and is followed by clear-cut post-ictal suppression; the recording of the seizure end-point is also free from any significant artefact. The deflections from about $1 \mathrm{~min} 21 \mathrm{~s}$ are artefact resulting from the ECT nurse cleaning electrode jelly from the scalp and the anaesthetist starting manual ventilation of the patient.

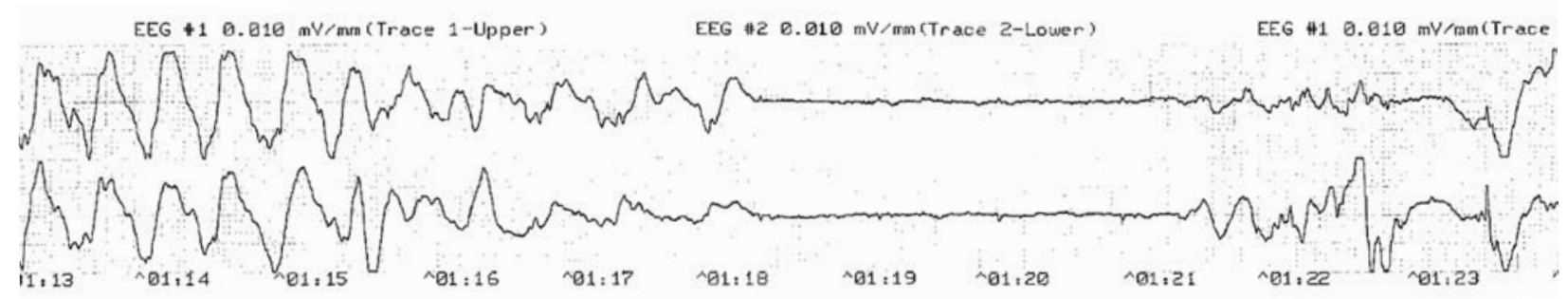

Fig. 1 An example of an EEG recording where the cerebral seizure ends abruptly and is followed by marked post-ictal suppression. 


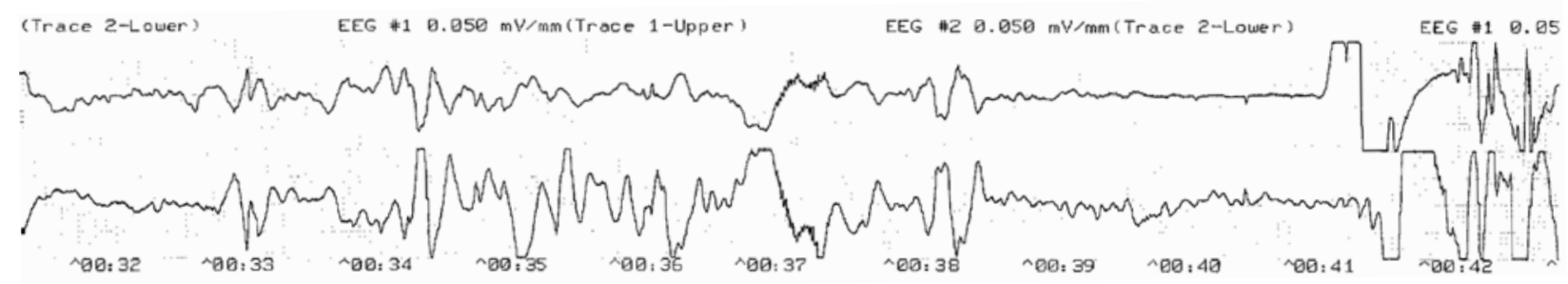

Fig. 2 An EEG recording where the termination phase is obscured by artefact (see text for details).
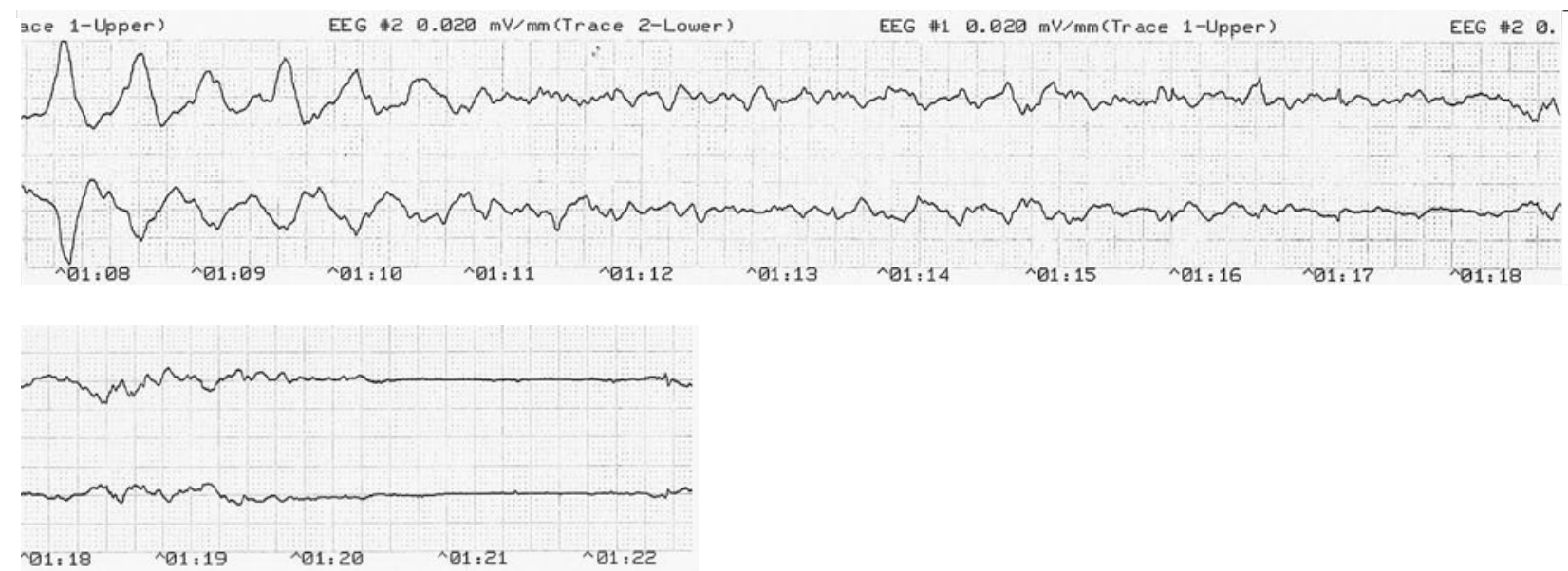

Fig. 3 Another EEG recording where the termination phase is obscured by artefact (see text for details).

In contrast, Fig. 2 shows an example of a recording of the termination phase in which the end-point of the cerebral seizure is almost totally obscured by artefact from the same sources as in Fig. 1. Nevertheless, there are about $2 \mathrm{~s}$ (between 39 and $41 \mathrm{~s}$ ) when the EEG recording is less affected by artefact, and it is possible to see that no clear-cut cerebral seizure activity is visible.

Figure 3 shows another recording of the termination phase in which the end-point of the cerebral seizure is obscured by artefact (associated with the manual ventilation of the patient). The first $3 \mathrm{~s}$ of the tracing in Fig. 3 show synchronous, slowwave activity at a frequency of about $2 \mathrm{~Hz}$, which is typical of the late ictal EEG. The anaesthetist had started manual ventilation of the patient before the end-point of cerebral seizure activity was seen. No clear-cut end-point was seen in the absence of the previous synchronous, slow-wave activity, and the anaesthetist was asked to remove the face mask at $1 \mathrm{~min} 20 \mathrm{~s}$; the EEG subsequently shows complete post-ictal suppression.

The tracing in Fig. 4(b) is an example of an EEG recording of a termination phase in which the determination of the end-point is difficult, not because of artefact, but because the degree of post-ictal suppression is insufficient to lead to a flat tracing after the cerebral seizure. A large-amplitude slowwave, just before $34 \mathrm{~s}$, is followed by only relative post-ictal suppression. The tracing in Fig. 4(a) was recorded just before electrical stimulation, and is included for comparison.

The impact of these practical problems is made worse when different practitioners try to resolve their determination of the end-point in different ways. There is no generally accepted convention in clinical practice, but Weiner and colleagues (1991) recommended that the end-point be taken as the most likely point at which the immediate post-ictal tracing begins. For example, in Fig. 2 it would be reasonable to conclude that the cerebral seizure has ended by $39 \mathrm{~s}$, although the exact end-point is obscured. It is not known whether the adoption of a standard approach to the determination of the end-point would reduce differences in the reported prevalences of prolonged cerebral seizure activity, but clearly this merits further study. Weiner and colleagues also noted that the lack of consensus about how to determine the cerebral seizure end-point might also contribute to differences between ECT clinics in the reported average length of cerebral seizures and how these relate to the length of convulsive activity. 


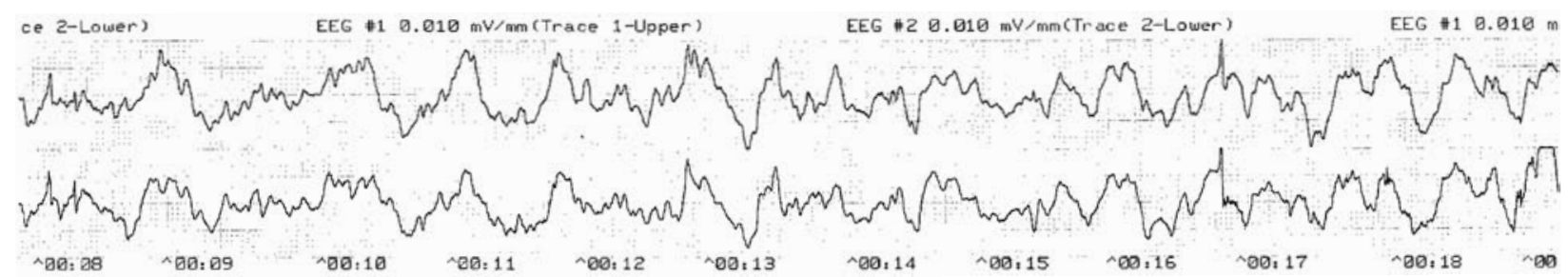

(a)

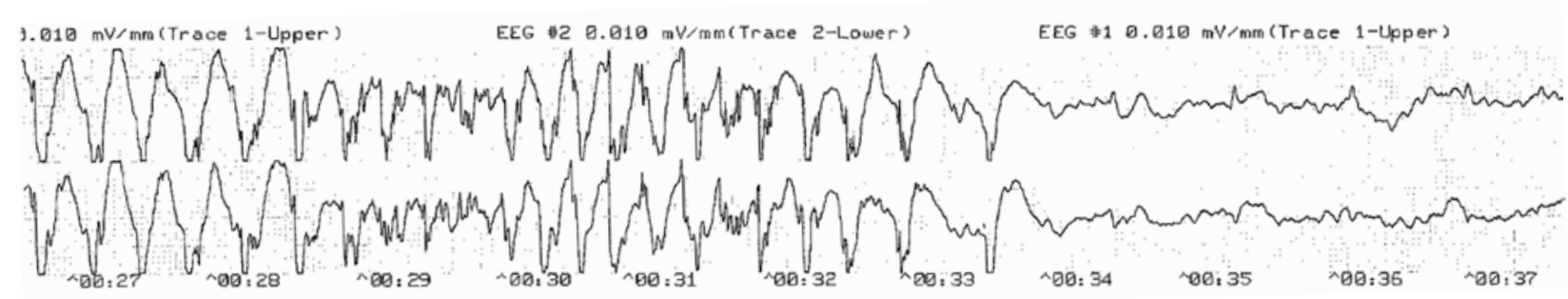

(b)

Fig. 4 Recording just before electrical stimulation (a), and resultant cerebral seizure followed by only relative post-ictal suppression (b).

\section{Utility of EEG monitoring}

Although EEG monitoring is the most direct available method of assessing cerebral seizure activity in the ECT clinic, there are clearly practical limitations to the use of this technique in routine clinical practice. Another aim of the Edinburgh study was therefore to assess the utility of EEG monitoring, in particular to measure the impact of difficulties in determining the end-point of cerebral seizure activity. Table 1 shows that a cerebral seizure of determinate length was obtained in only 93 out of 98 (95\%) first treatments in which a generalised convulsion was induced by threshold stimulation; in three cases cerebral seizure activity was observed on the EEG tracing, but the end-points could not be determined, and in two cases no cerebral seizure activity was observed. We had not anticipated that we would observe generalised convulsions in the absence of any detectable cerebral

$\begin{aligned} & \text { Table } 1 \text { EEG analysis made by clinic rater and inde- } \\ & \text { pendent neurophysiologist in } 98 \text { patients who experi- } \\ & \text { enced a generalised convulsion during the first ECT }\end{aligned}$
$\begin{aligned} & \text { Clinic rater } \\ & \text { No detectable } \\ & \text { cerebral seizure } \\ & \text { activity }\end{aligned}$
$\begin{aligned} & \text { Cerebral seizure of } \\ & \text { indeterminate length } \\ & \text { Cerebral seizure of } \\ & \text { determinate length }\end{aligned}$
$\begin{aligned} & \text { From Whittaker et al (2007). } \\ & \text { Nem }\end{aligned}$

seizure activity on the EEG, but it is clear from the literature that the brain is well insulated by the scull and scalp and that only a small proportion of cerebral seizure activity is detectable by surface EEG electrodes. This disassociation is more likely when electrical stimulation is close to the seizure threshold; in both cases, typical cerebral seizure activity was observed on the second treatment day, when the patients were treated with electrical stimulation $50 \%$ above the empirically determined seizure threshold.

It may have been hoped that the determination of the end-point of cerebral seizure activity would be made more reliable by the use of the computers built into contemporary ECT machines. Assessment of the reliability of these products may be a commercially sensitive topic. Over the past 10 years or so there have been only two studies of the reliability of computer-automated determinations of cerebral seizure end-points made by the type of ECT machine used in the Manchester study. Krystal \& Weiner (1995) found that the presence of any of the three problems mentioned above reduced the reliability of readings made both by computer-automated determination and by experienced ECT practitioners, but it was the computer-automated determinations that were particularly sensitive to the presence of these problems, particularly when they coexisted. Rosenquist et al (1998) subsequently reported that this system was unable to determine the cerebral seizure end-point in $28 \%$ of treatments.

The latest models of ECT machines have not been subject to such reliability studies. With this caveat, the hope that computers will determine the end-point more reliably than experienced ECT practitioners 
themselves has not yet been realised. Now that prompt medical treatment (intravenous induction agent or a benzodiazepine) is recommended for prolonged cerebral seizure activity, it no longer seems an appropriate aspiration that decisions about medical treatment potentially be delegated to a technology that cannot determine the seizure end-point in at least $5 \%$ of treatments.

\section{Practical tips for clinical practice}

\section{Timing of convulsion}

In any case, the revised College guidelines (Royal College of Psychiatrists, 2005) recommend that ECT clinics continue to monitor seizure activity by inspection and timing of the generalised convulsion because EEG monitoring is vulnerable to a number of technical problems: for example, tracings may be unreadable because of artefacts, connecting cables may become disconnected and the ECT machine may run out of paper unexpectedly. The findings discussed above show that there are more fundamental methodological problems that may compromise the utility of EEG recording, confirming the importance of monitoring by inspection of the convulsion as well as the increased use of the EEG.

\section{Preparing the patient and checking the apparatus}

Box 1 lists some practical tips for the preparation of the patient and for EEG recording. Although manufacturers of ECT machines can supply selfadhesive, pre-gelled EEG electrodes, preparation of the prefrontal and mastoid sites is still recommended to reduce electrical impedance. A clean with alcohol may be sufficient, but the use of a proprietary abrasive conductant gel may also be required (American Psychiatric Association, 2001). The quality of the recording should be checked while the patient is still

Box 1 Practical tips for EEG monitoring of ECT

- Prepare prefrontal and mastoid sites for EEG electrodes

- Inspect EEG recording while the patient is still awake, and make any necessary adjustments

- Record a few seconds of baseline EEG immediately before electrical stimulation

- 'Hands off' during the termination phase of EEG recording awake: are all channels working; is the amplification appropriate; is the tracing obscured by artefact? If the patient is still awake, there is still time to attend to any adjustments needed.

Incidentally, this is also a good learning opportunity for practitioners new to EEG recording. Asking the patient to open and close their eyes and move their eyeballs helps to familiarise the ECT practitioner with the appearance of movement artefacts: it will soon be appreciated that deflections resulting from muscle movements easily swamp electrical activity attributable to the brain itself.

\section{Analysing the termination phase}

A record of the baseline tracing can prove helpful in the analysis of the termination phase if the cerebral seizure is followed by only relative post-ictal suppression. It cannot be assumed that a readable EEG tracing of the termination phase can be obtained if the patient's head is touched or moved by the treatment room staff. 'Hands-off' assistance by nursing and anaesthetic colleagues will be necessary if EEG monitoring of the termination phase is to be practicable. (This is not such a pressing problem during the polyspike and slow-wave phase because the amplitude of electrical activity is at its peak.)

\section{Determining the seizure end-point}

The following may be of practical help if it is difficult to determine the seizure end-point. In the Edinburgh study (Whittaker et al, 2007) the only patient who required medical treatment to terminate a prolonged cerebral seizure detectable by EEG had also displayed a prolonged generalised convulsion, that is, a generalised convulsion lasting more than $90 \mathrm{~s}$. Even if the EEG is not useful in determining whether or not cerebral seizure activity is ended, there may be other signs that this is so: the anaesthetist may report that the patient is starting to breathe spontaneously or show other signs of recovering from anaesthesia. Such observations argue against the presence of cerebral seizure activity. If the seizure end-point cannot be determined from the EEG and the patient shows no clinical signs of recovery of consciousness, medical intervention may be entirely appropriate.

\section{EEG monitoring in the assessment of seizure adequacy}

Over and above the hope that EEG monitoring would be a direct measure of whether or not the necessary cerebral seizure activity had been induced and had ended was the hope that it would also inform the 
Box 2 Potential clinical applications of the ictal EEG, beyond measurement of the length of cerebral seizure activity

- Inform prescription of optimal electrode placement

- Inform prescription of optimal electrical dose

- Predict eventual treatment response

- Inform adjustment of electrical dose over a course of treatment

- Provide data to commercial computerautomated algorithm to determine seizure adequacy

Adapted from Mayur (2006)

ECT practitioner about whether or not this activity was of a quality that would maximise the clinical efficacy of ECT.

The full analysis of an EEG recording as would be conducted by a neurophysiologist is a complex matter, but the ECT literature contains comprehensive reviews that are approachable by the interested ECT practitioner (Krystal \& Weiner, 1999; Nobler et al, 2000).

The clinical utility of EEG has been recently reviewed by Mayur (2006), who surveyed the literature up to October 2005 on the ictal EEG in ECT. The potential clinical applications of EEG monitoring, beyond confirmation of the start and end-point of cerebral seizure activity, are listed in Box 2. Monitoring can detect statistically significant differences between ECT techniques of differing efficacy, for example unilateral and bilateral electrode placement and high- and low-dose treatment. It is yet to be shown, however, that any of these statistically significant differences is of a sufficient magnitude to provide clinically useful guidelines for the ECT practitioner.

This can be illustrated by reference to one of the key studies of the association between the degree of post-ictal suppression and clinical improvement in symptoms of depression (Suppes et al, 1996). Although this study found a statistically significant correlation between the average degree of post-ictal suppression after the first six treatments in a course of ECT and the extent of improvement in symptoms of depression, inspection of the raw data shows that the correlation is not sufficiently close to provide a robust prediction of clinical improvement by inspection of the post-ictal suppression. Thirteen patients with depression experienced at least a $60 \%$ reduction in depressive symptoms over the first six treatments, but the average degree of post-ictal suppression ranged from almost none to complete.
Mayur (2006) found no studies of the predictive accuracy that relied solely on computer-automated algorithms available in contemporary ECT machines, and this too may be a commercially sensitive topic. Mayur concluded that the development of such algorithms was the next logical step for clinical practice, but made no comment at all about the commercial algorithms presently available.

It therefore remains the case that after the necessary cerebral seizure activity has been induced and recorded by EEG, it is not yet possible for the ECT practitioner to examine an individual EEG tracing and make an accurate prediction about clinical outcome after a course of treatment. Nevertheless, EEG monitoring can contribute to the treatment of individual patients during a course of ECT. If the patient has not experienced significant clinical improvement and the EEG recording does not show robust and/or typical cerebral seizure activity, this may contribute to the decision to either increase the electrical dose or switch from unilateral to bilateral electrode placement. It remains to be shown that commercial computer-automated determinations of seizure adequacy contribute to these decisions.

\section{Declaration of interest}

A.S. is the editor of the 2nd edition of The ECT Handbook (Royal College of Psychiatrists, 2005).

\section{References}

American Psychiatric Association (2001) The Practice of Electroconvulsive Therapy: Recommendations for Treatment, Training and Privileging (2nd edn). APA.

Benbow, S. M., Benbow, J. \& Tomenson, B. (2003) Electroconvulsive therapy clinics in the United Kingdom should routinely monitor electroencephalographic seizures. Journal of ECT, 19, 217-220.

Krystal A. D. \& Weiner, R. D. (1995) ECT seizure duration: reliability of manual and computer-automated terminations. Convulsive Therapy, 11, 158-169.

Krystal A. D. \& Weiner, R. D. (1999) EEG correlates of response to ECT: a possible antidepressant role of brain-derived neurotropic factor. Journal of ECT, 15, 27-38.

Mayur, P. M. (2006) Ictal electroencephalographic characteristics during electroconvulsive therapy: a review of determination and clinical relevance. Journal of ECT, 22, 213-217.

Mayur, P. M., Gangadhar, B. N., Janakiramaiah, M., et al (1999) Motor seizure monitoring during electroconvulsive therapy. British Journal of Psychiatry, 174, 270-272.

Nobler, N. S., Luber, B., Moeller, J. R., et al (2000) Quantitative EEG during seizures induced by electroconvulsive therapy: relations to treatment modality and clinical features. I. global analyses. Journal of ECT, 16, 211-228.

Rosenquist, P. B., McCall, W. V., Collenda, C. C., et al (1998) A comparison of visual and computer-generated measures of "seizure quality". Journal of ECT, 14, 76-82.

Royal College of Psychiatrists (2005) The ECT Handbook. Second Edition. The Third Report of the Royal College of Psychiatrists' Special Committee on ECT (Council Report CR128). Royal College of Psychiatrists.

Scott, A. I. F. (2005) College guidelines on electroconvulsive therapy: an update for prescribers. Advances in Psychiatric Treatment, 11, 150-156. 
Suppes, T., Webb, A., Carmody, T., et al (1996) Post ictal electrical silence: a predictor of response to electroconvulsive therapy? Journal of Affective Disorders, 41, 55-58.

Weiner, R. D., Coffey, C. E. \& Krystal, A. D. (1991) The monitoring and management of electrically induced seizures. Psychiatric Clinics of North America, 14, 845-869.

Whittaker, R., Scott A. \& Gardner, M. (2007) The prevalence of prolonged cerebral seizures at the first treatment in a course of electroconvulsive therapy. Journal of ECT, 23, 11-13.

\section{MCQs}

1 The following contribute to difficulty in determination of the end-point of cerebral seizure activity:

a too low an impedance at the electrode sites

$b$ overzealous preparation of the sites for EEG electrodes

c abrupt end to cerebral seizure activity

d complete post-ictal suppression

e prompt manual ventilation by the anaesthetist after the generalised convulsion ends.

2 In the analysis of an EEG recording where the endpoint is obscured by artefact, the end-point should be taken as:

a end of observed polyspike and slow-wave activity

b the last observable spike wave

c the last observable slow wave

$\mathrm{d}$ the starting point of post-ictal baseline

e 20 s after the last clear-cut slow wave.

3 When the ECT practitioner has difficulty determining the end-point of a cerebral seizure on an EEG recording, the following are unlikely to help diagnose the presence or absence of a prolonged seizure:

a output from the ECT machine's in-built computer

$b$ the length of generalised convulsive activity
C the ordinal number of the treatment in the course of ECT

d the return of spontaneous respiration

e purposeful movement by the patient.

4 The Royal College of Psychiatrists recommends that cerebral seizure activity should be medically terminated after:

a $60 \mathrm{~s}$

b $90 \mathrm{~s}$

c $120 \mathrm{~s}$

d $150 \mathrm{~s}$

e $180 \mathrm{~s}$

5 In ECT with stimulus dosing, the quality of the ictal EEG is significantly related to:

a the patient's gender

b the patient's psychiatric diagnoses

c the absolute electrical dose

d electrode placement

e a course of ECT 4 months before index treatment.

\section{MCQ answers}

\begin{tabular}{|c|c|c|c|c|}
\hline 1 & 2 & 3 & 4 & 5 \\
\hline a $F$ & a $F$ & a $\mathrm{T}$ & a $F$ & a $F$ \\
\hline $\mathrm{bF}$ & b F & b F & b F & b F \\
\hline c F & c F & c F & c $\mathrm{T}$ & c $\mathrm{F}$ \\
\hline $\mathrm{d} F$ & $\mathrm{~d} T$ & d F & $\mathrm{d} F$ & $\mathrm{~d} T$ \\
\hline e $\mathrm{T}$ & e $\mathrm{F}$ & e F & e $F$ & e $F$ \\
\hline
\end{tabular}

